\title{
Cognitive Alterations in Elderly Patients with Heart Failure'
}

\author{
Fernanda Guedes Afiune, ${ }^{1 \text { (1) }}$ Salvador Rassi, ${ }^{10}$ Abrahão Afiune Neto ${ }^{2}$ (을 \\ Universidade Federal de Goiás, ' Goiás, GO - Brazil \\ Sociedade Brasileira de Cardiologia, ${ }^{2}$ Rio de Janeiro, RJ - Brazil
}

\section{Abstract}

Background: Among the various pathologies that affect the elderly, Heart Failure (HF) stands out. Recently, an attempt has been made to verify the existence of cognitive impairment associated with HF.

Objectives: To compare the cognitive performance of elderly people with heart failure with that of age-matched individuals without this pathology. Check the existence of marked impairment in some cognitive functions in the clinical group.

Methods: The sample consisted of 78 elderly people, whose inclusion criterion was the presence of HF and no HF (control group); age over 60 years, both sexes, and any level of education. The control group consisted of 37 individuals (with a median age of 68 years - Interquartile range of 12) and the HF group, with 41 individuals (with a median age of 67 years - Interquartile range of 11). The subjects were matched in terms of education level, with a predominance of elderly people with 0 to 4 years of education $(65.9 \%$ in the Clinical Group and $59.5 \%$ in the Control Group). Eleven neuropsychological tests covering cognitive functions were used: attention, language, memory, mood, and executive function. Statistical analysis was performed using SPSS software, version 23, with a significance level of 5\%. The Chi-square test and the Mann-Whitney test were applied.

Results: The results showed significant differences between the groups, mainly in executive functions, which include the ability to plan, switch, and recall previously stored information.

Conclusion: Our study showed differences between the cognitive performance of elderly people with HF and elderly people without HF. The main alteration was found in the so-called executive functions, attention, and memory.

Keywords: Heart Failure; Neuropsychology; Cognition Disorders; Aged; Cardiovascular Diseases; Neurological Diseases; Anxiety; Depression; Stroke Volume.

\section{Introduction}

With the increasing size of the elderly population worldwide, the concern about the quality of life in the face of aging has been a continual subject of study. ${ }^{1-3}$ Among the most prevalent pathologies in the elderly, cardiovascular and neurological diseases stand out the most, with Heart Failure (HF) being the number one cause of hospitalizations, morbidity, and mortality, amongst other cardiovascular diseases. ${ }^{4}$
It is well-known that HF, described as a progressive disease caused by heart muscle damage, is commonly associated with physical alterations, functional loss, and even emotional alterations, which contribute to the diminishing of one's quality of life. ${ }^{5}$ More recently, however, the understanding of the cognitive changes caused by this pathology has been the main focus of study. The etiology of the cognitive changes in cardiovascular diseases (HF among them) remains unknown, however, the majority of studies confer two

Mailing Address: Fernanda Guedes Afiune

R. 235, s/n. Postal Code: 74605-020, Setor Leste Universitário, Goiânia, GO - Brazil.

E-mail: fernanda_guedesa@hotmail.com 
physiological processes, which can explain the presence of cognitive impairment in patients with HF that could be the hypoperfusion of the brain and multiple cardiogenic emboli. $^{6-7}$

The literature points out that HF affects various aspects of cognitive functioning, including attention, learning ability, memory (specifically evocation), working memory, executive function, and psychomotor speed, ${ }^{8-9}$ while language and visual-spatial ability seem to be less investigated aspects. ${ }^{10}$

Memory, on the other hand, is one of the most researched and studied cognitive functions, primarily in the elderly, perhaps due to it being the biggest complaint among patients of this age. ${ }^{11}$ Mnemonic development in HF individuals has also been indicated in most studies, pointing to short and long-term verbal memory impairment. ${ }^{12-14}$ Episodic memory changes are noted in HF patients above the age of $80{ }^{8}$

Nevertheless, few references mention the executive functions, which are those responsible for the ability to control and monitor human behavior, mental flexibility, decision-making, and problem-solving. ${ }^{15}$ Discoveries made in the change of these functions are still recent14. Mood swings due to the presence of such symptoms as anxiety and depression can also be found in the elderly. ${ }^{16-18}$

Therefore, the present study aimed to compare the general cognitive performance in elderly patients with HF with elderly individuals from the control group (without HF) through neuropsychological tests, as well as to verify if there are losses in any specific functions of HF patients.

\section{Method}

This is a descriptive-comparative cross-sectional study between two groups of elderly individuals, which was performed through neuropsychological testing of members of the community and patients of the HF emergency ward of a renowned hospital in the city of Goiania. The protocol (CAAE:44041414.4.0000.5078) was approved by the Ethics Committee of the Hospital das Clínicas of Federal University of Goiás.

\section{Characterization of the studied group}

Using a significance level of $5.0 \%$ and a test power of $80 \%$, with a margin of error of $7 \%$, the sample consisted of 78 elderly people, whose inclusion criteria were the presence of HF and without HF (control group - CG). Both groups consisted of subjects over 60 years of age, both sexes, and with any level of education. Subjects included those with the loss of ability to understand or express speech (aphasia), the presence of motor impairment in the dominant upper limb that would make graphic production unfeasible, the presence of sequelae caused by a cerebrovascular accident/stroke or traumatic brain injury, and the diagnosis of neurological and/or psychiatric disorders, such as dementia.

The HF group was set up in the HF Emergency Ward after reviewing patients' medical records to verify each participant's inclusion criteria in the research. The control group, in turn, was set up using the non-probabilistic approach, with the search performed for convenience (made in the community at random according to the subject's availability and respecting the study inclusion criteria). As regards the sociodemographic data, the vast majority of the patients were of a low socioeconomic level. The HF group was completely comprised of individuals who received outpatient care from the Brazilian public health system: "Unified Health System" (SUS). The control group was paired with individuals with similar socioeconomic levels and similar levels of education.

The HF group was classified according to the type of HF presented, those being: Preserved Ejection Fraction (HFpEF), Reduced ejection fraction (HFrEF), and Ejection Fraction "borderline" (EF "borderline"). It was found that $51.2 \%$ of the individuals of the HF group presented Reduced EF (LVEF $\leq 40 \%), 29 . .3 \%$ presented preserved EF (LVEF $\geq 50 \%$ ) and $19.5 \%$ of the HF group sample had EF "borderline" (LVEF=40\% to 49\%). All data were obtained from the patients' medical records.

The evaluator was aware during the test of which group each individual belonged to.

All participants signed the "free and informed consent form" (TCLE) before the beginning of the study.

\section{Instruments and procedure}

All study subjects underwent neuropsychological tests in a single session of approximately 50 minutes and were evaluated by the sameevaluator. The session time followed the pattern of time of a neuropsychological session (time needed to apply different testing instruments). The tests were applied in the following sequence: Rey Auditory Verbal Learning Test (Part A1 to A5, B1, and A6); ${ }^{19}$ Rey Complex Figure Test; ${ }^{20}$ Wechsler Adult Intelligence Scale Subtest (Direct Order and Reverse Order); ${ }^{21}$ The Rey Osterrieth Complex Figure; ${ }^{20}$ Trail Making Test (Form A), ${ }^{22}$ Rey Auditory Verbal Learning Test (Part A7 
and Recognition);19 Phonemic Verbal Fluency (FAS);22 Semantic Verbal Fluency (Animals); ${ }^{22}$ Beck Anxiety Scale, and Beck Depression Scale. ${ }^{23}$

The results were collected and obtained through an initial interview (data collection of individual identification and socioeconomic level), medical report analysis (comorbidities and ejection fraction data from the HF group), and the application of the tests mentioned above.

\section{Statistical analysis}

This study chose to use non-parametric tests, since it focused on the field of study of cognition, whose dimensions are not considered to be corresponding to the normal population distribution.

The Statistical Package for the Social Sciences (SPSS) software, version 23, was used to conduct statistical analyses, and a significance level of $5 \%$ was adopted for all statistical tests. The data in the tables are presented with the average and the interquartile range.

Pearson's Chi-Square test was applied to compare the socio-demographic variables, sex, and education. Mann-Whitney's test was used to compare the age variable between the groups as well as the differences found between the sexes (by comparing the average of the clinical and control groups subdivided by genre). The same test was used to compare the performance between the groups in each instrument applied in this study.

\section{Results}

\section{Demographic characteristics of the sample}

The Control Group consisted of 37 individuals, 10 of whom were male and 27 female. No individuals from this group presented any diagnosed cardiac, neurological, or psychiatric pathology. In turn, the HF group consisted of 41 individuals, of whom 20 were male and 21 female, all of whom presented Symptomatic HF and were under outpatient medical follow-up. The socio-demographic data of these groups are presented in Table 1.

As significant differences were observed between the sexes in the studied groups (HF and CG), as seen in Table 1, a comparison of performance in each neuropsychological test applied between the sexes of each group was carried out separately. Each group analysis was conducted and no statistically significant differences were noted between the men and women of each group. This analysis allows us to conclude that the difference between genders (male

Table 1 - Demographic data of the studied groups - gender, education, and age variables

\begin{tabular}{|c|c|c|c|c|c|}
\hline & \multicolumn{2}{|c|}{ HF } & \multicolumn{2}{|c|}{$\mathrm{CG}$} & \multirow[b]{2}{*}{$p$} \\
\hline & $\mathbf{n}$ & $\%$ & $\mathbf{N}$ & $\%$ & \\
\hline \multicolumn{6}{|l|}{ Gender } \\
\hline Male & 20 & 48.8 & 10 & 27.0 & \multirow{2}{*}{0.04} \\
\hline Female & 21 & 51.2 & 27 & 73.0 & \\
\hline \multicolumn{6}{|l|}{ Education } \\
\hline 0 to 4 years & 27 & $65.9 \%$ & 22 & $59.5 \%$ & \\
\hline 5 to 8 years & 7 & $17.1 \%$ & 9 & $24.3 \%$ & \\
\hline 9 to 11 years & 1 & $2.4 \%$ & 4 & $10.8 \%$ & 0.26 \\
\hline > 12 years & 6 & $14.6 \%$ & 2 & $5.4 \%$ & \\
\hline Age & \multicolumn{2}{|c|}{ HF } & \multicolumn{2}{|c|}{ CG } & $p$ \\
\hline AV (IQ) & \multicolumn{2}{|c|}{$67(11)$} & \multicolumn{2}{|c|}{$68(12)$} & 0.93 \\
\hline
\end{tabular}


and female) observed in the HF and CG groups (with a predominance of females in the control group) did not interfere in the final analysis regarding the cognitive performance of each group.

\section{Statistical analysis of clinical and control groups regarding performance on neuropsychological tests}

The results regarding the performance in the neuropsychological tests performed in this study will be presented according to the main function that the test intends to evaluate. In this way, the tests were grouped according to the following neuropsychological functions: attention and executive function (Table 2), language and mood (Table 3), and memory (Table 4).

\section{Discussion}

No differences between the groups regarding socioeconomic demographics (age and education) were identified. However, there was a statistical difference between the sexes in the evaluated groups, with the clinical group having a greater number of elderly males than the control group. The difference between the sexes did not interfere in the final analysis of the results, because when comparing the average performance in the tests performed by men and women in each group, no statistical differences were observed between them. Several studies, in turn, have already pointed out that there is no difference between the neuropsychological performance of men and women. ${ }^{24-28}$

With regard to attention capacity, statistical differences were found between the groups. The analysis conducted in this study showed difficulties concerning alternate attention observed through the Reverse Order Digits test.

Along with the assessment of attention, the assessment of mental processing speed was also performed. Mental processing speed and attention are functions commonly assessed in individuals with HF when compared to controls or controls with heart disease..$^{8,13-14}$

The data found here showed a significant difference between the groups in this aspect, which was observed through the Trail Making Test, form A. When analyzing the performance between the groups, it was noticed that the worst performance occurred in the HF Group, which showed a delay in the processing speed when compared to the CG. This data is similar to the data found in other studies, which report impairment in psychomotor/mental speed in individuals with HF. 4,7,12,14,29-31 Therefore, this analysis allows us to take into account that individuals with HF have slow mental processing, which is compatible with all the symptoms presented by this disease.

When referring to the executive function, it is important to emphasize that this domain encompasses different skills: planning, decision-making, impulse

Table 2 - Statistical analyses between the studied groups for neuropsychological function (NPF) of attention and executive function

\begin{tabular}{|c|c|c|c|c|}
\hline \multirow[b]{2}{*}{ NPF } & \multirow[b]{2}{*}{ Tests } & \multicolumn{2}{|r|}{ CG } & \multirow[b]{2}{*}{$p$} \\
\hline & & AV (IQ) & AV (IQ) & \\
\hline \multirow{6}{*}{ Attention } & TMT A & $14.00(102)$ & $91.00(62)$ & 0.05 \\
\hline & SD Total & $9.0(5)$ & $10.00(2)$ & 0.09 \\
\hline & DDO & $5.00(2)$ & $6.00(2)$ & 0.19 \\
\hline & DRO & $3.00(2)$ & $4.00(2)$ & 0.05 \\
\hline & UDIOD & $4.00(1)$ & $4.00(1)$ & 0.15 \\
\hline & UDIOI & $3.00(1)$ & $3.00(1)$ & 0.005 \\
\hline \multirow{2}{*}{ Executive Function } & DOI & $3.00(2)$ & $4.00(2)$ & 0.05 \\
\hline & $\mathrm{RCF}$ & $17.5(17)$ & $27.50(9)$ & 0.001 \\
\hline
\end{tabular}


Table 3 - Statistical analysis between the studied groups for neuropsychological function (NPF) of language and mood

\begin{tabular}{lcccc}
\hline \multirow{2}{*}{ FNP } & & HF & CG & \\
\cline { 3 - 5 } \multicolumn{1}{c}{ Language } & Tests & Md (IQ) & Md (IQ) & p \\
\hline \multirow{2}{*}{ Mood } & FAS & $18.0(16)$ & $23.00(12)$ & $\mathbf{0 . 0 3}$ \\
\cline { 2 - 5 } & LF Animals & $11.0(5)$ & $12.00(6)$ & $\mathbf{0 . 3 2}$ \\
\hline & BAI & $11.0(16)$ & $13.00(15)$ & $\mathbf{0 . 8 2}$ \\
\hline
\end{tabular}

Md: Median; IQ: Interquartile Range; FAS: Phonemic Verbal Fluency; LF Animals - Semantic Verbal Fluency -Animal Category; BAI: Back Anxiety Inventory; BDI: Beck Depression Inventory; p: Mann-Whitney

Table 4 - Statistical analysis between the studied groups for neuropsychological function (NPF) of memory

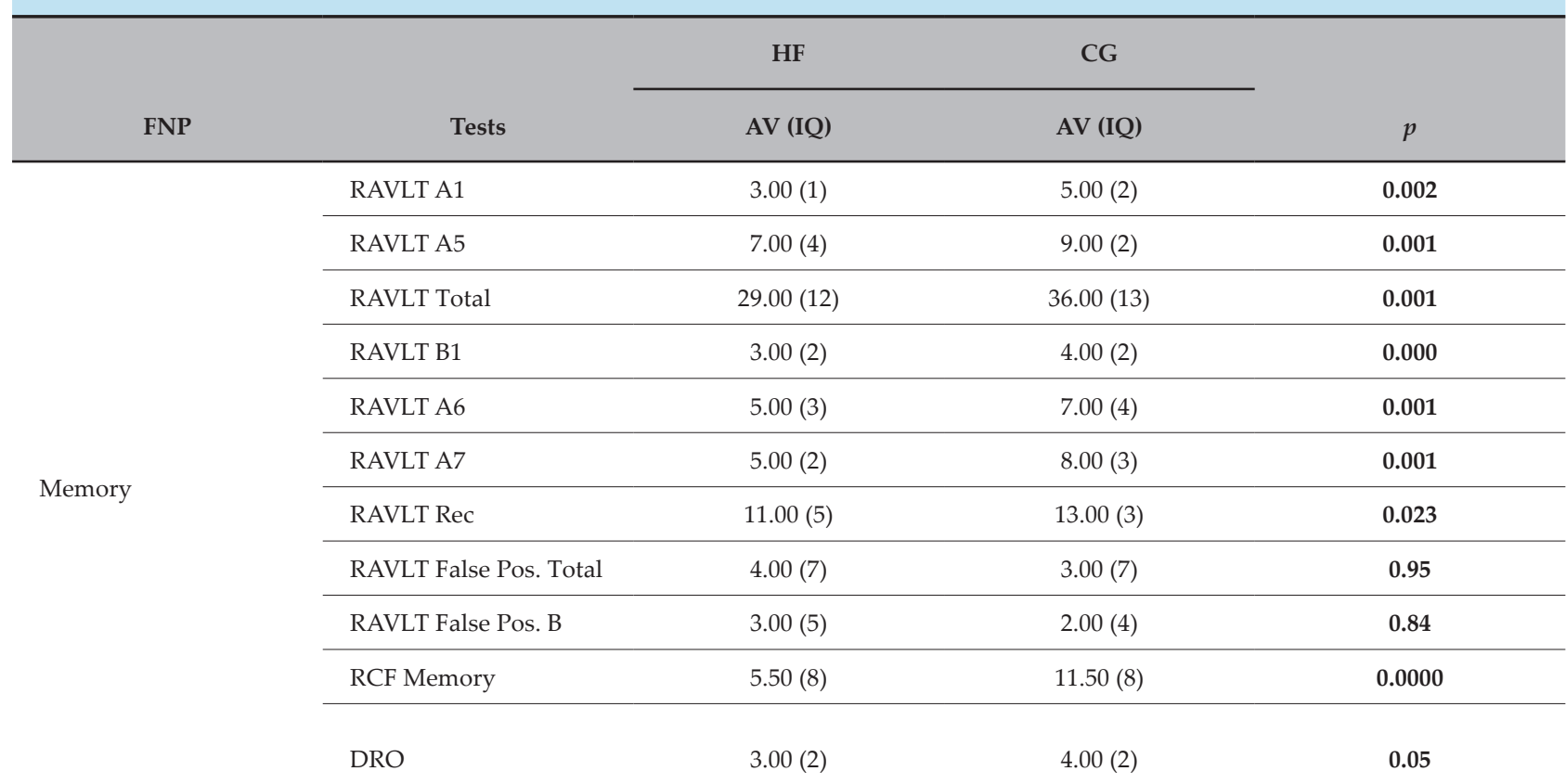

AV: Average; IQ: Interquartile Range; RAVLT: Rey Auditory Verbal Learning Test; A-List A; Total-total responses obtained in the five presentations on list A; B-interference list; Rec- Recognition of List A items through multiple choice presentation; False Pos.intrusions committed in recognition tasks; RCF-Rey Complex Figure; DRO: Digits Reverse Order; UDIOD: Number of digits repeated in direct order: UDIOI: Number of digits repeated in reverse order; $p$ : Mann-Whitney

control, organization, working memory, alternation, and self-regulation. The present study evaluated the alternation and planning abilities. The data showed a difference between the groups, revealing the worst performance for the group of patients with HF in terms of planning and organization abilities, assessed using the Rey's Complex Figure Copy (RCF) test. Patients in the HF group presented a significantly lower performance than what was obtained from the CG, which indicated difficulty in planning and organizing an activity with a higher level of mental demand, since the test is comprised of a complex geometric figure with many details.

Alternation ability and mental flexibility were evaluated using the Indirect Order Digits test, with a significant difference between the groups in this skill. 
The impairment of executive functions has been indicated and evidence has been verified in different

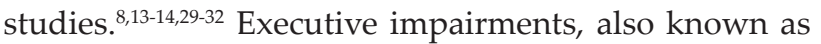
executive dysfunction, are commonly associated with reduced instrumental activities, both leisure and social, as well as difficulty performing daily routine activities in HF patients. Discrepant performances regarding language domain were observed between the groups through the assessment of the Phonetic Verbal Fluency (PVF) test; however, in the Semantic Verbal Fluency (Animals), no difference was found in performance when comparing both groups.

It is worth noting that the verbal fluency test is a test that suffers significant interference from the processing speed (the individual must reproduce words of a letter or semantic category in a pre-established time of 1 minute) and executive function (the search for information is performed by this area of the brain, more specifically by the frontal lobe). Considering, therefore, that language ability, specifically assessed by verbal fluency tests, suffers interference from these two domains (processing speed and executive function), and that most studies point out impairments in these cognitive domains in HF individuals, verbal fluency may have been altered as a result of the losses of other domains (executive and processing speed). This, therefore, is the justification for the poor performance of patients in the HF group in the phonemic verbal fluency test. Thus, it can be said that the primary impairment of patients with $\mathrm{HF}$ is in the executive functions and processing speed; however, they can also interfere in the performance of other cognitive functions, as occurred in this study when language was assessed.

The present study opted to analyze and evaluate the presence of symptoms of depression and anxiety in the elderly population, although it is observed that anxiety is rarely reported and studied in this population when compared to depression. No differences were found between the evaluated groups as regards the presence of symptoms of depression and anxiety. However, when analyzing the average obtained by the two groups studied, on both the Beck Depression Scale and the Beck Anxiety Scale, both groups presented results considered to be in the mild range, that is, there is a mild presence of symptoms of depression and anxiety, as set forth by the Portuguese Version of the Manual of the Beck Scales. ${ }^{22}$ These data demonstrated that as many CG individuals as those with HF presented mild mood impairments (anxiety and depression), despite not showing statistical differences between them.
These data corroborate the results found by Vogels et al. ${ }^{14}$ who pointed out similar results with symptoms of depression and anxiety in individuals with HF, individuals with heart disease, and individuals in the control group (with healthy aging). Studies only with individuals with HF also report the presence of symptoms of depression in this population, ${ }^{18,30,32}$ which are associated with decreased cognition and, consequently, greater functional dependence and less participation in society.

The high prevalence of symptoms of depression in the elderly population in Brazil has been confirmed in many studies. ${ }^{33-34}$ Aspects related to the perception of health itself (negative perception and fear of falls, diseases, among others) are an important factor associated with the presence of depressive symptoms in the elderly. The perception of health is a subjective fact, and it refers to how the person sees their general condition, including biological, psychological, and social factors. ${ }^{33}$ Hence, taking into account the current situation in Brazil, both in terms of health care and how the elderly are treated by society, we can justify that the similar results presented by the groups in the assessment of mood are most likely due to the perception that the elderly have concerning their health and future in Brazil, whether in the presence of an established pathology or not.

It is noteworthy that most studies conducted to assess mood aspects use self-report instruments that depend on the individual's self-assessment and self-perception, which can interfere with the real prevalence of these factors.

Memory is the main complaint and concern brought up by the elderly population. In this study, the mnemonic performance for auditory and visual stimuli was chosen to be evaluated, together with working memory. The assessment of auditory mnemonic performance was performed using the Rey Auditory-Verbal Learning Test. Concerning short-term auditory memory (RAVLT A1), a difference was observed between the evaluated groups. This data suggests that short-term auditory memory has a worse performance in HF when compared to CG. During the test, the stimuli continued to be presented, and the individual was asked to recall each attempt (new presentation of the stimuli) so that we could verify the learning capacity when faced with the new content. It was observed that the groups showed a significant difference amongst themselves at the end of the learning curve (RAVLT A5). The IC group showed worse performance when compared to the control group, 
despite demonstrating benefits in terms of repetition for learning, qualitatively observed by an increasing learning curve. The low performance presented by the HF group may have suffered interference from the mental fatigue presented throughout the testing situation. Thus, it can be stated that short-term auditory memory in patients with HF differed from the control group, corroborating Sauvé's study. ${ }^{31}$

Still analyzing the auditory mnemonic performance, the IC group showed a worse performance than did the CG when a new list of words to be memorized was presented (RAVLT B1) and when they should evoke the previously memorized words after the interference (RAVLT A6). The presentation of a new list (B1) assesses the individual's ability to resist the effect of proactive distractors, that is, the interference of previously learned content on the learning of new content. ${ }^{35}$ The impairment presented between the groups can be explained by the difficulty in alternating, which is characteristic of impairment in the executive function. The difficulty of switching was observed in this study in individuals with $\mathrm{HF}$ and has been highlighted above. This means that when faced with a new list (List B), individuals with HF demonstrated difficulty in returning to the first list (List A), which had been previously presented.

Long-term auditory memory (RAVLT A7) was also worse in patients with HF, as well as in the face of cues for recollection (RAVLT Rec). Data analysis allowed us to identify that the two studied groups showed impairment in information retrieval, which refers to impairment associated with frontal alterations (related to executive functions). It is known that many memory changes in the elderly are related to the loss of frontal lobe functions, a region that is extremely vulnerable to aging, both structurally and functionally. ${ }^{36}$ This means that the HF group, as well as the CG, can store the information presented, but cannot retrieve it spontaneously, needing clues and tips due to predominantly frontal alterations.

For the short-term visual memory assessment, the Rey's Complex Figure test was used, which is one of the most commonly used neuropsychological tests in clinical practice to assess visual memory and some functions of planning and executing actions. ${ }^{37}$ The HF group performed worse than the CG in this skill. The low impairment of visual memory occurred as a result of the difficulty in planning during copying, which interfered with its subsequent recall. Executive functions, which are associated with frontal areas, have, among other functions, the function of planning and organizing information and actions. It was observed that patients with HF had a worse performance in copying the Rey Complex Figure when compared to CG due to a change in executive performance (specifically in planning). Thus, the poor performance presented in memory is related to executive impairment. Oliveira and Rigoni ${ }^{20}$ confirm this information by stating that the better the organization and planning during the execution of the copy of the figure, the better its recall.

Working memory, which refers to the temporary storage and manipulation of information in a short period of time ${ }^{38}$ was also evaluated in this study through the performance in the Reverse Order Digits subtest. The number of numbers that individuals can repeat in reverse order (UDIOI) was also evaluated. The working memory ability differed between the evaluated groups, differing from the data found by Pressler et al. ${ }^{30}$ in their study.

The data found in this study clashes with some previously conducted studies when evaluating the memory capacity in patients with HF. This can occur due to the different instruments used and the fact that most studies refer only to the total performance value in the memory test, not considering the different types of memory and stages of this process, which are: encoding, consolidation, storage, and recovery. ${ }^{7,23,38}$

Another factor that can justify the discrepancy found refers to the fact that, in the elderly, the decrease in mnemonic performance may not be attributed to a memory alteration itself. It is known that, in elderly persons, other factors interfere in the mnemonic process, such as a slow processing speed (which can directly or indirectly interfere with the processing time of complex sequences/operations), as well as the reduction of attentional resources available for cognitive processing (the reduction of attention associated with age interferes with the elderly person's ability to carry out cognitively demanding processes, such as coding operations and the elaboration of information retrieval strategies, which are important in the learning process). Furthermore, the elderly have deficits in inhibitory control associated with age. Inhibitory control is important for memory, as it controls access to working memory by delimiting access to relevant information, as well as supporting the inhibition of information overload and controlling possible situationally inappropriate responses. Allied to these factors are impairments in the executive control of the elderly. In this case, we are referring to automatic processes (which require little attention span and occur without intention) and controlled processes (which 
are those that require focused attention as well as the intention to carry out the activity). Automatic processing is unaltered in the aging process, while the controlled processing declines with age. ${ }^{11}$ Thus, it can be concluded that in individuals with HF, memory and learning capacity were impaired; however, other cognitive impairments (attention and executive functions) interfere in the process of consolidation, acquisition, and recall of short-term and long-term information.

Considering all the data discussed in this study, the importance of neuropsychological assessment in elderly patients with HF was noted. The assessment becomes important since cognitive impairment in these individuals can compromise the ability to recognize the worsening of HF symptoms and the ability to make appropriate decisions about their own health. In addition, cognitive impairments generate difficulty in drug management, which increases the risk of decompensation of HF, hospitalizations, and even death. It is also known that the association between cognitive deficits in HF directly interferes with one's quality of life. ${ }^{8,13-14}$

As regards the limitations of the study, it should be highlighted that this study did not compare the presence of brain alterations measured through imaging exams with the identified cognitive alterations. Such a comparison could explain which factors could be related to cognitive alterations, allowing for a better prognosis of the condition. Another limitation of the study was that it did not compare the HF functional class in the HF group, the presence of fragility, the presence of Chronic Obstructive Pulmonary Disease (COPD), and which drugs were being used in this same group (HF). Thus, it was not possible to verify whether there is a direct relationship between greater severity of the disease and worse cognitive impairment. Another key limiting factor is the fact that the evaluator was not blinded in

\section{References}

1. Campos ACV, Vargas AMD, Ferreira EF. Active Aging Determinant According to Quality of Life and Gender. Ciências e Saúde Coletiva 2015; 20(7): 2221-37.

2. Cosenza RM, Malloy-Diniz L. Healthy Aging, Cognitive Resilience and Quality of Life. In: Malloy-Diniz L. (Org.). Neuropsychology of Aging - a multidimensional approach. Porto Alegre: Artmed; 2013. Cap. 26, p. 438-46.

3. Tahan J Carvalho ACD. "Reflections from participating elderly in health-promoting groups regarding aging and quality of life". Saúde e Sociedade. 2010; 19(4): 878-88

4. Alosco ML,Spitznagel MB, Cohen R, Sweet LH, Josephson R, Hudghes $\mathrm{J}$, et al. Cognitive deficits are associated with poorer simulated driving in older adults with heart failure. BMC Geriatrics. 2013; 13:58. this study and was aware of which group each elderly person belonged to during the application of the tests.

\section{CONCLUSIONS}

The present study showed evidence of the differences between the cognitive performance of elderly individuals with heart failure and elderly individuals who are free of this pathology. The main identified changes were in the so-called executive functions and attention, these brain functions being associated with the prefrontal and frontal regions. The changes in memory previously shown in other studies have also been noted in this research.

\section{Potential Conflict of Interest}

No potential conflict of interest relevant to this article was reported.

\section{Sources of Funding}

The current study was financed by the responsible researcher herself.

\section{Study Association}

This article is part of the thesis of master submitted by Fernanda Guedes Afiune, from Universidade Federal de Goiás.

\section{Ethics approval and consent to participate}

This study was approved by the Ethics Committee of the Hospital das Clínicas -Universidade Federal de Goiás under the protocol number 44041414.4.0000.5078. All the procedures in this study were in accordance with the 1975 Helsinki Declaration, updated in 2013. Informed consent was obtained from all participants included in the study.

5. Ayub-Ferreira SM. Physiopathology of Heart Failure In: Moreira MCV, Montenegro ST, Paola AAV (Eds.). Livro-texto da Sociedade Brasileira de Cardiologia. 2. ed. Barueri, SP: Manole; 2015. Seção 17, cap. 3, p. 1116-67.

6. Jesus PAP, Vieira de Melo R, Reis F, Viana L, Lacerda A, Dias JS, et al. Congestive Dysfunction in congestive heart failure: transcranial Doppler evidence of microembolic etiology. Arq Neuropsiq. 2006 jun;64(2):207-10.

7. Vogels RLC, Sheltens P, Schroeder JM, Winster HC.Cognitive impairment in heart failure: a systematic review of the literature. Eur Heart Fail.2007;9(5):440-9.

8. Abete P, Morte DL, Gargiulo G, Basile C, Langelloto A, Galizia G, et al. Cognitive impairment and cardiovascular diseases in the elderly. A heart-brain continuum hypothesis. Ageing Res Rev. 2014 Aug 11; 18 : 41-52. 
9. Bennett SJ, Sauvé MJ. Cognitive deficits in patients with heart failure: a review of the literature. J. Cardiovasc. Nurs. 2003;18(3):219-42.

10. Bauer LC, Johnson JK, Pozehl, BJ. Cognition in heart failure: an overview of the concepts and their measures. J Am Acad Nurse Pract .2011; 23(11): 577-85.

11. Abrisqueta-Gomez J. Memory and healthy cognitive aging. In: MalloyDiniz LF, Fuentes D, Cosenza RM (Org.). Neuropsychology of aging - a multidimensional approach. Porto Alegre: Artmed; 2013. Cap. 10, p. 171-96.

12. Almeida OP, Garrido GJ, Beer C, Lautsen Schlager NT, Flicker AL. Cognitive and brain changes associated with ischaemic heart disease and heart failure. Eur Heart J. 2012 Jul; 33(14): 1769-76.

13. Alosco ML,Spitznagel M, Cohen R, Raz N, Swat LH, Josephscen R, et al. Reduced cerebral perfusion predicts greater depressive symptoms and cognitive dysfunction at a 1-year follow-up in patients with heart failure. Int J Geriatr Psychiatry. 2014; 29:428-36.

14. Vogels RLC,Dosterman JM, van Horten B, ScheltensP, Flier V, SchroederTanka J, et al. Profile of cognitive impairment in Chronic Heart Failu Am J Geriatr Soc;2007;55(11):1764-70.

15. Uehara E, Mata F, Fichman HC, Malloy-Diniz LF. Executive Functions in Childhood. In: Salles JF, Haase VG, Malloy-Diniz LF (Org.). Neuropsychology of development - childhood and adolescence. Porto Alegre: Artmed; 2016. Cap. 1, p. 17-2

16. Almeida OP, Beer C, Lautenschlager NT, Arnolda L, Alfonso H, Flicker $\mathrm{L}$, et al. Two-year course of cognitive function and mood in adults with congestive heart failure and coronary artery disease: the Heart-Mind Study. Int Psychogeriatr.2012; 24(1):38-47.

17. Almeida OP,Garrido G, Etherton-Beer C, Laustenschlanger NT, Arnolda $\mathrm{L}$, Alfonso $\mathrm{H}$, et al. Brain and mood changes over 2 years in healthy controls and adults with heart failure and ischaemic heart disease. Eur J Heart Fail. 2013;15(8):850-8.

18. Steinberg G Lossnitzer N, Schellberg D, Mueller Tasch T, Krueger C,et al. Peak Oxygen uptake and left ventricular ejection fraction, but not depressive symptoms, are associated with cognitive impairment in patients with chronic heart failure. Int J Gen Med. 2011;4.2011;4:879-87.

19. Wechsler D. Wechsler Adult Intelligence Scale Manual - WAIS III. São Paulo: Editora Casa do Psicólogo; 2004.

20. Oliveira MS, Rigoni MS. The Rey Osterrieth Complex Figure. ... quires the subject to copy, and later reproduce from memory São Paulo: Casa do Psicólogo; 2010.

21. Strauss E, Sherman EMS, Spreen O. A compendium of Neuropsychological Tests. Oxford: Oxford University Press; 2006.

22. Cunha JÁ. "Beck Scales Manual Portuguese Version" São Paulo: Casa do Psicólogo; 2001.

23. Homes $\mathrm{O}$ (Org.). Prevalence of Memory Disorders in Ambulatory Patients aged $\geq 70$ with Chronic Heart Failure. Am J Cardiol.2014;113(7):1205-10.
24. Zibetti MR .“Comparative study of neuropsychological functions between the ages of 21 and 90 years of age." Rev Neuropsicol Lat Am. 2010; 2(1): 55-67.

25. Foss MP, Bastos-Formighieri MS, Speciali JG. Rey 's Complex Figures for the Elderly. "Psychol Evaluat. 2010;8(1):53-61.

26. Soares E, Coelho MO, Carvalho SMR. Functioning ability, cognitive decline and depression in institutionalized elderly: possibilities of relations and correlations Revista Kairós Gerontologia 2012 set; 15(5):117-39.

27. Martins MR et al. "Alternative versions of Logical Memory subtest of WMS-R: performance analysis of a healthy sample of São Paulo". Psicologia, Reflexão e Crítica 2015; 28(3): 444-53.

28. Magalhães SS, Hamdan AC. The Rey Auditory Verbal Learning Test: normative data for the Brazilian population and analysis of the influence of demographic variables. Psychol and Neurosc.2010;3(1):85-90..

29. Dardiotis E,Glamouzis G, Mastrogiannis D, Voglatzi C, Skoulariguido Iriposkiaadis, et al.. Cognitive impairment in heart failure. Cardiol Res Pract. 2012; 2012: 1-9

30. Pressler SJ.SubPerkens SM, Ramanian U, Kareken D,Perkens SM, Gradus -PC et al. Cognitive deficits in chronic heart failure. Nurs Res. 2010; 59(2):127-39.

31. Sauvé SMJ,Subramanian U,Levis W, Bankslibbe, M,Rickabaigh B, Pressler S, et al.. Cognitive impairments in chronic heart failure: a casecontrolled study. J Card Fail. 2009;15(1):1-10.

32. Foster ER, Executive dysfunction and depressive symptoms associated with reduced participation of people with severe congestive heart failure. Am J Occup.2011;65(3):306-13.

33. Ramos GCF,Ramos MA, Bansal N, Khan R, Guille C.. Prevalence of Depressive Symptoms and Associated Factors in the Elderly in the North of Minas Gerais: a Population-Based Study. J Bras Psiquitr. 2015; 64(2):122-31.

34. Carneiro AM, Baptista MN, Santos AAA. Measures of depressive symptoms in the elderly: Sociodemographic variables relations. Avances en Psicología Latinoamericana 2013; 31(3): 483-92.

35. Cotta MF Rey Auditory Verbal Learning Test (RAVLT) in the differential diagnosis of normal and pathological cognitive aging. Clin Cont. 2012; $5(1): 10-25$.

36. Cosenza RM. "Memory and its changes during aging - normal and pathological". In: Tavares A. (Org.). Compêndio de Neuropsiquiatria Geriátrica. Rio de Janeiro-RJ: Guanabara Koogan; 2005. Cap.12, p. 145-56.

37. Gazzaniga MS, Ivry RB, Mangun GR. Cognitive Neuroscience - Mind Approach Porto Alegre: Artmed; 2006.

38. Kandel ER. (Org.). Principles of Neuroscience Porto Alegre: AMGH; 2014. 